\title{
IDEAL TYPES IN A POLYNOMIAL HALFRING
}

\author{
LOUIS DALE
}

\begin{abstract}
Ideal types are used to classify ideals in a polynomial halfring and to determine when an ideal behaves like a $k$-ideal. In particular, these results are used to classify all ideals in the ring of polynomials over the integers.
\end{abstract}

1. Introduction. The type of a $k$-ideal $K$ in a halfring $H$ has been defined to be the set of all $k$-ideals $I$ in the ring of differences $\bar{H}$ such that $I \cap H=K$. Using $\tau(K)$ to denote the ideal type of $K$, an ideal is said to be monotypic if $\tau(K)$ consists of a single ideal. While the concept of ideal type is applicable to $k$-ideals in any halfring, it is of interest to consider ideal types in polynomial halfrings. The purpose of this paper is to consider a special ideal type found only in polynomial halfrings. The general concept of ideal type is extended to include all ideals in a halfring and an attempt is made at relating ideal types in a halfring $H$ to ideal types in $H[x]$.

2. Ideal types in $H$ and $H[x]$. A semiring is a set $S$ together with two binary operations called addition $(+)$ and multiplication $(\cdot)$ such that $(S,+)$ is an abelian semigroup with a zero, $(S, \cdot)$ is a semigroup and multiplication distributes over addition from both the left and the right. If $(S,+)$ is also cancellative, then $S$ is called a halfring. A halfring $S$ is said to be strict if $a, b \in S$ and $a+b=0$ imply $a=b=0$. An ideal $I$ in a semiring $S$ is called a $k$-ideal if $a \in I, b \in S$ and $a+b \in I$ imply $b \in I$. The subtractive ideals mentioned in [4] and $k$-ideals have the same meaning. Now every ideal $A$ in a semiring $S$ is contained in a unique $k$-ideal $\bar{A}_{\kappa}$, called the $k$-closure of $A$. This fact allows one to extend the definition of ideal type as follows:

2.1. Definition. Let $H$ be a halfring and $A$ an ideal in $H$. The ideal type of $A$, denoted $\tau(A)$, is the set of all ideals $I$ in the ring of differences $\bar{H}$ such that $I \cap H=\bar{A}_{\kappa}$.

Since $\bar{A}_{\kappa}$ is a $k$-ideal in $H$, it is clear that $\tau(A)=\tau\left(\bar{A}_{\kappa}\right)$ for any $A$ in $H$.

Stone [4] used the type of an ideal to relate halfring ideals to ideals in the ring of differences. Thus it is easy to see why the type of an ideal is totally dependent on ideals in the ring of differences and is defined only for $k$-ideals. Now every ideal in a halfring is not a $k$-ideal. However, some ideals behave

\footnotetext{
Received by the editors April 5, 1978 and, in revised form, July 14, 1978 and November 10, 1978.

AMS (MOS) subject classifications (1970). Primary 16-02, 16A78; Secondary 16A66.

Key words and phrases. Semiring, halfring, ideal type, monotypic, $k$-ideal, weak $k$-ideal, $k$-closure.
} 
more like $k$-ideals than others. To investigate such ideals it seems natural to consider ideals in a polynomial halfring, since the degree of a polynomial could possibly aid in the investigation. The fact that these ideals are not $k$-ideals suggests that the concept of ideal type in the usual sense would be of no help. Consequently, it appears that an ideal type is needed that is independent of ideals in the ring of differences and defined for all ideals in the halfring.

Throughout this paper, unless otherwise stated, $H$ will be a commutative halfring with an identity and $H[x]$ will be the halfring of polynomials over $H$ in the indeterminate $x$. An ideal $A$ in $H[x]$ is called a weak $k$-ideal if there exists an integer $n$ such that $A$ is a $k$-ideal with respect to all polynomials $f \in A$ with degree $f \leqslant n$. The largest such integer if it exists is called the $k$-degree of $A$. If no such integer exists, then $A$ is said to have $k$-degree $\infty$.

2.2. Definition. Let $A$ be an ideal in $H[x]$. The ideal type of $A$ in $H[x]$, denoted $\tau_{0}(A)$, is the set of all weak $k$-ideals $I$ in $H[x]$ such that $A \subset I \subset \bar{A}_{\kappa}$.

It is easy to see that $\tau_{0}(A)$ is closed under unions of chains and arbitrary intersections, and contains $\bar{A}_{\kappa}$ as a maximal element. Thus, $\tau_{0}(A)$ is never empty.

Now there are associated two ideal types with each ideal $A$ in $H[x], \tau(A)$ and $\tau_{0}(A)$, a set of ideals in $\bar{H}[x]$ and a set of ideals in $H[x]$ respectively. The importance of the ideal type $\tau(K)$ of a $k$-ideal $K$ in a halfring $H$ lies in its relation to the collection of homomorphic images of $H$ having $K$ as kernel. The ideal type $\tau_{0}(A)$ is important because ideals in $\tau_{0}(A)$ can be used to investigate ideals in $H[x]$ that behave like $k$-ideals.

Let $A$ be an ideal in $H[x], n$ a fixed integer and

$$
A_{f_{n}}=\{f \mid f \in A \text { and degree } f \leqslant n\} .
$$

The ideal $\bar{A}_{\kappa_{n}}=\bigcap\{B \mid B$ is a weak $k$-ideal with $k$-degree at least $n$ and $\left.A_{f_{n}} \subset B\right\}$ is called the weak $k$-closure of $A$.

It is clear that $\bar{A}_{\kappa_{n}}$ has $k$-degree at least $n$ and $A_{f_{n}} \subset \bar{A}_{\kappa_{n}} \subset \bar{A}_{\kappa}$. Also, if $m<n$, then it follows that $\bar{A}_{\kappa_{m}} \subset \bar{A}_{\kappa_{n}}$. Consequently, with each ideal $A$ in $\underline{H}[x]$ there is associated an ascending chain of weak $k$-ideals $\left\{\bar{A}_{\kappa_{n}}\right\}$ such that $\bar{A}_{\kappa_{n}} \subset \bar{A}_{\kappa}$ for each nonnegative integer $n$. Now if $H$ is a strict halfring and $W_{i}=A+\bar{A}_{\kappa_{i}}$, then $\left\{W_{i}\right\}$ is an ascending chain of ideals in $\tau_{0}(A)$ such that the $k$-degree of $W_{i}$ is at least $i$. To see that $W_{i} \in \tau_{0}(A)$, observe that $A_{f_{i}} \subset \bar{A}_{\kappa_{i}}$ and $\bar{A}_{\kappa_{i}}$ is a weak $k$-ideal with $k$-degree at least $i$. Since $H$ is strict, it follows that $f \in W_{i}$ and $\operatorname{deg} f \leqslant i$ imply $f \in \bar{A}_{\kappa_{i}}$. Consequently, $W_{i}=A+\bar{A}_{\kappa_{i}}$ is a $k$-ideal with respect to all polynomials of degree less than or equal to $i$ and it follows that $W_{i}$ is a weak $k$-ideal with $k$-degree at least $i$. Now suppose $I \in \tau_{0}(A)$. If the $k$-degree of $I$ is $\infty$, then $I=\bar{A}_{\kappa}$ and it follows that $W_{i} \subset I$ for all $i$. If $I$ has finite $k$-degree, say $t$, then it is clear that $\bar{A}_{k_{1}} \subset I$ and consequently, $W_{t}=A+\bar{A}_{\kappa_{1}} \subset I$. This proves the following:

2.3. LeMma. If $H$ is a strict halfring, $A$ is an ideal in $H[x]$, and $I \in \tau_{0}(A)$, then there exists $i$ such that $W_{i} \subset I$. 
This lemma establishes that the ideals $\left\{W_{i}\right\}$ serve as sort of a basis for $\tau_{0}(A)$. Consequently, with each nonzero ideal $A$ in $H[x]$ there can be associated a unique number $\omega=\left|\left\{W_{i}\right\}\right|$.

2.4. Definition. An ideal $A$ in $H[x]$ will be called a quasi-k-ideal if $\omega$ is finite.

Since $W_{i}=A+\bar{A}_{\kappa_{i}}$, it is clear that the number $\omega$ associated with $A$ is really the number of distinct weak $k$-closures of $A$. Consequently, $\omega$ tells us the minimum number of steps required to construct $\bar{A}_{\kappa}$ using weak $k$-ideals. Thus the $k$-closures of quasi $k$-ideals are easier to construct than for others.

It is clear that if $H[x]$ is a Noetherian halfring, then every ideal in $H[x]$ is a quasi- $k$-ideal. However, requiring that $H[x]$ be Noetherian is a strong condition to impose. This can be weakened somewhat by requiring that $H$ be Noetherian. It was shown in [1] that $H$ is Noetherian if and only if every ascending chain of monic ideals in $H[x]$ is finite. An ideal $M$ in $H[x]$ is called monic if $\sum a_{i} x^{i} \in M$ implies that $a_{i} x^{i} \in M$ for each $i \in$ $\{0,1,2, \ldots, n\}$.

2.5. THEOREM. If $H$ is a Noetherian halfring, then every monic ideal in $H[x]$ is a quasi-k-ideal.

Proof. Let $A$ be a monic ideal and $W_{n} \in \tau_{0}(A)$. Then it follows that $\bar{A}_{\kappa}$ is a monic ideal. To see this, note that the $k$-boundary of $A$ is

$$
A^{\prime}=\{g \in H[x] \mid \text { there is } f \in A \text { such that } f+g \in A\} .
$$

It was shown in [1] that $\overline{A_{\kappa}}=A^{\prime}$. Let $g=\sum b_{i} x^{i} \in \overline{A_{\kappa}}$. Then there exists a polynomial $f=\sum a_{i} x^{i} \in A$ such that

$$
f+g=\Sigma\left(a_{i}+b_{i}\right) x^{i} \in A .
$$

It could happen that $f=0$. Since $A$ is monic, $\left(a_{i}+b_{i}\right) x^{i}=a_{i} x^{i}+b_{i} x^{i} \in A$ for each $i$. Now $a_{i} x^{i} \in A$ and it follows that $b_{i} x^{i} \in \bar{A}_{\kappa}$ and $\bar{A}_{\kappa}$ is monic. Now let $\mathfrak{T}$ be the set of all polynomials in $\bar{A}_{\kappa}$ with degree at most $n$. By a proof similar to the above, it follows that $\vec{A}_{\kappa_{n}}$ is a monic ideal with respect to polynomials in $\mathfrak{K}$. If $M=(\mathfrak{K})$ is the ideal generated by $\mathfrak{N}$, then $M$ is a monic ideal,

$$
A_{f_{n}} \subset \Re \subset M \subset \bar{A}_{\kappa_{n}}
$$

and the $k$-degree of $M$ is at least $n$. Consequently, $M=\bar{A}_{\kappa_{n}}$ and $\overline{A_{\kappa_{n}}}$ is a monic ideal. Hence, $W_{n}=A+\bar{A}_{\kappa_{n}}$ is a monic ideal. Therefore $\left\{W_{i}\right\}$ is an ascending chain of monic ideals in $H[x]$ and must necessarily be finite. Consequently, $\omega$ is finite and $A$ is a quasi $k$-ideal.

Since $Z^{+}$is a Noetherian halfring, it is clear that every monic ideal in $Z^{+}[x]$ is a quasi- $k$-ideal. However, there are quasi- $k$-ideals in $Z^{+}[x]$ that are not monic, as the following example will show.

2.6. Examples. (i) Consider the ideal $A=\left(a, x^{n}+a\right)$ in $Z^{+}[x]$, where $a>1$ and $n \geq 1$. Now $A$ is neither a monic ideal nor a $k$-ideal since $x^{n} \notin A$. 
Any polynomial of degree $m<n$ in $A$ is of the form $\sum c_{i}\left(a x^{i}\right)$ and it follows that $A$ is a $k$-ideal with respect to these polynomials since each $c_{i}\left(a x^{i}\right) \in A$. Consequently, $A$ is a weak $k$-ideal of degree $n-1$. From this it follows that

$$
\bar{A}_{\kappa_{1}}=\bar{A}_{\kappa_{2}}=\cdots=\bar{A}_{\kappa_{n-1}}=A
$$

and hence $W_{1}=W_{2}=\cdots=W_{n-1}$. Now any $k$-ideal containing $a$ and $x^{n}+a$ must contain $x^{n}$. Since $\left(a, x^{n}\right)$ is a $k$-ideal, it is clear that $\bar{A}_{k_{n}}=$ $\left(a, x^{n}\right)$. This gives $W_{n}=\bar{A}_{\kappa_{n}}$ and consequently, $W_{n}=W_{n+1}=\cdots$. Therefore $\left|\left\{W_{i}\right\}\right|=\omega=2$ and $A$ is a quasi- $k$-ideal.

(ii) Consider the ideal $B=(x+1)$ in $Z^{+}[x]$. Any $k$-ideal, say $K$, containing $x+1$ must also contain $x^{3}+1$. For

$$
(x+1)^{3}=x^{3}+1+3 x(x+1) \in K
$$

and $3 x(x+1) \in K$ imply that $x^{3}+1 \in K$. By induction, any $k$-ideal containing $x+1$ must also contain $x^{2 n+1}+1$ for each $n \in Z^{+}$. Let $B_{0}=(x$ $+1), B_{n}=\left(x^{2 n+1}+1\right)+B_{n-1}$ if $n \geqslant 1$, and $E=\cup B_{n}$. Then $\left\{B_{i}\right\}$ is an ascending chain of ideals in $Z^{+}[x]$ and $E$ is an ideal such that $E \subset B_{\kappa}$. Now let $\eta: Z^{+}[x] \rightarrow Z$ be defined by $\eta(f(x))=f(-1)$. It can be shown directly that $\eta$ is a semiring homomorphism and $\operatorname{ker} \eta=E$. Consequently, $E$ is a $k$-ideal containing $B$ and it follows that $\bar{B}_{\kappa} \subset E$. This shows that $E=\bar{B}_{\kappa}$. Since $E$ is a $k$-ideal it follows that for any positive integer $n$ the set $E_{f_{n}}$ of all polynomials in $E$ with degree at most $n$ generates a weak $k$-ideal whose $k$-degree is at least $n$. Hence $\bar{E}_{\kappa_{n}} \subset\left(E_{f_{n}}\right)$. Now $E_{f_{n}} \subset \bar{E}_{\kappa_{n}}$ and it follows that $\left(E_{f_{n}}\right) \subset \bar{E}_{\kappa_{n}}$. Consequently, $\bar{E}_{\kappa_{n}}=\left(E_{f_{n}}\right)$, i.e., the weak $k$-closure of degree $n$ of a $k$-ideal is generated by $E_{f_{n}}$. Now note that the set $\left\{x^{2 n+1}+1 \mid n \in Z^{+}\right\}$is a basis for $E$. Clearly $E_{f_{1}} \subset B_{0}=(x+1)$ and it follows that $\bar{E}_{\kappa_{1}}=B_{0}$. Also, $E_{f_{2}} \subset B_{0}$ since the basis elements in the set $\left\{x^{2 n+1}+1 \mid n>0\right\}$ cannot generate polynomials of degree 2 . Consequently, $\bar{E}_{\kappa_{2}}=B_{0}=\bar{E}_{\kappa_{1}}$ and it follows that $W_{1}=W_{2}$. Now $E_{f_{3}}, E_{f_{4}} \subset B_{1}=B_{0}+\left(x^{3}+1\right)$ since the basis elements in the set $\left\{x^{2 n+1}+1 \mid n>1\right\}$ cannot generate polynomials of degree less than 5. Consequently, $\bar{E}_{\kappa_{3}}=\bar{E}_{\kappa_{4}}=B_{1}$ and it follows that $W_{3}=W_{4}$. From $B_{0} \neq B_{1}$ it follows that $\bar{E}_{\kappa_{2}} \neq \bar{E}_{\kappa_{3}}$ and consequently $W_{2} \neq W_{3}$. Continuing in this manner, one obtains the sequence of ideals $\left\{W_{i}\right\}$ with the property that for each $i=2 n+1, W_{i}=W_{i+1}$ but $W_{i} \neq W_{i+2}$. Therefore $\left|\left\{W_{i}\right\}\right|=\omega=\infty$ and $B$ is not a quasi- $k$-ideal.

These two examples illustrate the difference in the construction of the $k$-closure of a quasi- $k$-ideal and the $k$-closure of an ideal that is not a quasi- $k$-ideal.

3. Ideal classes in $H$ [ $x$ ]. It is of interest to find some relation between ideal types in $H$ and ideal types in $H[x]$. For the collection of ideals in $H[x]$ of the form $E[x], E$ an ideal in $H$, this is an easy matter. For if $I$ is an ideal in $\bar{H}$, then $I \cap H=E$ if and only if $I[x] \cap H[x]=E[x]$. Consequently, $I \in$ $\tau(E)$ if and only if $I[x] \in \tau(E[x])$ and it follows that the map $I \rightarrow I[x]$ is a 
lattice isomorphism between $\tau(E)$ and a subset of $\tau(E[x])$. Now with each nonzero ideal $A$ in $H[x]$ there is associated an ascending chain of ideals $\left\{A_{i}\right\}$ in $H$, where

$$
A_{i}=\left\{a \in H \mid \text { there is an } f \in A \text { such that } a x^{i} \text { is a term of } f\right\} .
$$

These ideals are called coefficient ideals, and it is clear that $\cup A_{i}=A^{\prime}$ is an ideal in $H$. Consequently, each ideal $A$ in $H[x]$ can be associated with a unique ideal in $H$, namely $A^{\prime}$. If $\mathcal{H}$ is the collection of all ideals in $H$ and $\mathcal{G}$ the collection of all ideals in $H[x]$, then the map $\mu: \mathcal{G} \rightarrow \mathcal{H}$ given by $\mu(A)=A^{\prime}$ is surjective. Define a relation " $\sim$ " in $\mathcal{G}$ as follows; $A \sim B$ if and only if $A^{\prime}=B^{\prime}$, or equivalently, $A \sim B$ if and only if $A, B \in \mu^{-1}\left(C^{\prime}\right)$ for some $C^{\prime} \in \mathcal{H}$. It is clear that this is an equivalence relation and consequently partitions $\mathcal{G}$ into equivalence classes. Note that if $\alpha$ is an equivalence class of $\mathcal{G}$ and $A \in \alpha$, then $\alpha=\mu^{-1}\left(A^{\prime}\right)$. Thus the equivalence classes are induced by $\mu$. If $\mathcal{G}^{*}$ is the set of equivalence classes of $\mathcal{G}$, then the map $\mu^{*}: \mathcal{H} \rightarrow \mathcal{G} *$ given by $\mu^{*}\left(A^{\prime}\right)=\alpha$, where $\alpha$ is the equivalence class containing $A$, is bijective. For if $\alpha \in \mathcal{G}^{*}$ and $A \in \alpha$, then $\alpha=\mu^{-1}\left(A^{\prime}\right)$ and it follows that $\mu^{*}\left(A^{\prime}\right)=\alpha$. If $\mu^{*}\left(A^{\prime}\right)=\mu^{*}\left(B^{\prime}\right)$, then

$$
\alpha=\mu^{-1}\left(A^{\prime}\right)=\mu^{-1}\left(B^{\prime}\right)=\beta
$$

and it follows that $A \sim B$. Consequently, $A^{\prime}=B^{\prime}$ and $\mu^{*}$ is bijective. This proves the following.

3.1. THEOREM. Let $H$ be $a$ halfring with an identity, $\mathcal{H}$ the collection of all ideals in $H$ and $\mathcal{G}$ the collection of all ideals in $H[x]$. The map $\mu: \mathcal{G} \rightarrow \mathcal{H}$ given by $\mu(A)=A^{\prime}$ induces an equivalence relation on $\mathcal{G}$. Moreover, there is a one-to-one correspondence between $\mathcal{H}$ and the set of equivalence classes of $\mathcal{G}$.

It is clear that this result can be extended from the halfring $H$ to its rings of differences $\bar{H}$.

3.2. Corollary. Let $H$ be $a$ halfring with an identity and $\bar{H}$ its ring of differences. If $\overline{\mathcal{H}}$ is the collection of all ideals in $\bar{H}$ and $\overline{\mathcal{G}}$ the collection of all ideals in $\bar{H}[x]$, then the map $\bar{\mu}: \overline{\mathcal{G}} \rightarrow \overline{\mathcal{H}}$ given by $\bar{\mu}(A)=A^{\prime}$ induces an equivalence relation on $\overline{\mathcal{G}}$. Moreover, there is a one-to-one correspondence between $\overline{\mathcal{H}}$ and the set of equivalence classes of $\overline{\mathcal{G}}$.

The preceding theorem establishes a correspondence between the ideals in $H$ and certain classes of ideals in $H[x]$. Now to establish a relation between ideals in $H$ and $\bar{H}$ as well as $H[x]$ and $\bar{H}[x]$.

3.3. LEMMA. If $A$ is an ideal in $H[x]$, then $\bar{\mu}(\bar{A})=\overline{\mu(A)}$.

Proof. Since $\bar{\mu}(\bar{A})=(\bar{A})^{\prime}$ and $\overline{\mu(A)}=\overline{A^{\prime}}$ it suffices to show that $(\bar{A})^{\prime}=\overline{A^{\prime}}$. Suppose $c \in(\bar{A})^{\prime}$. Then $c \in(\bar{A})$, for some $t$ and it follows that $c x^{t}$ is a term of $f-g$, where $f=\sum a_{i} x^{i}, g=\sum b_{i} x^{i} \in A$. Thus

$$
c x^{t}=\left(a_{t}-b_{t}\right) x^{t}
$$


and consequently, $c=a_{t}-b_{t} \in \overline{A_{t}} \subset \overline{A^{\prime}}$. On the other hand, if $c \in \overline{A^{\prime}}$, then $c=a-b$, where $a, b \in A^{\prime}=\cup A_{i}$. Thus $a \in A_{j}, b \in A_{k}$ for some integers $j$ and $k$. Since $\left\{A_{i}\right\}$ is an ascending chain either $A_{j} \subset A_{k}$ or $A_{k} \subset A_{j}$; say $A_{k} \subset A_{j}$. There exists polynomials $f, g \in A$ such that $a x^{j}$ and $b x^{j}$ is a term of $f$ and $g$ respectively. Consequently, $c x^{j}=a x^{j}-b x^{j}$ is a term of $f-g \in \bar{A}$ and it follows that $c \in(\bar{A})_{j} \subset(\bar{A})^{\prime}$. Therefore $(\bar{A})^{\prime}=\bar{A}^{\prime}$.

If $A$ is an ideal in $H[x]$, then $\bar{A}$ is an ideal in $\bar{H}[x]$. Consequently, $\mu(A)=A^{\prime}$ is an ideal in $H$ and $\mu(\bar{A})=(\bar{A})^{\prime}$ is an ideal in $\bar{H}$. Since $A \in$ $\mu^{-1}\left(A^{\prime}\right)=\alpha, \bar{A} \in \bar{\mu}^{-1}(\bar{A})^{\prime}$ and $(\bar{A})^{\prime}=\bar{A}^{\prime}$, let $\bar{\alpha}=\bar{\mu}^{-1}\left(\overline{A^{\prime}}\right)$. Then it is clear that $B \in \alpha$ implies $\bar{B} \in \bar{\alpha}$.

3.4. Definition. The ideal type of the class $\alpha=\mu^{-1}\left(A^{\prime}\right) \in G^{*}$, denoted $\tau(\alpha)$, is the set of all classes $\beta$ such that $\beta=\bar{\mu}^{-1}\left(B^{\prime}\right)$ for some $B^{\prime} \in \tau\left(A^{\prime}\right)$.

Note that in order for the above definition to make sense, it is necessary that $\tau\left(A^{\prime}\right)$ be defined for all ideals in $H$. Consequently, we made use here of the extended definition of ideal type.

Let $\alpha=\mu^{-1}\left(A^{\prime}\right) \in G^{*}$ and consider the ideal $\overline{A^{\prime}}$ in $\bar{H}$. Now $\overline{A^{\prime}} \cap H=E$ is a $k$-ideal in $H$ containing $A^{\prime}$. If we denote the $k$-closure of $A^{\prime}$ by $K$, then it is clear that $K \subset E$. If $x \in E$, then $x \in H$ and $x \in \overline{A^{\prime}}$ and it follows that $x=a-b$ for some $a, b \in A^{\prime}$. Thus $a=b+x$ and $x \in K$. Consequently, $E \subset K$ and it follows that $E=K$. Therefore, $\overline{A^{\prime}} \cap H=K$ and $\overline{A^{\prime}} \in \tau\left(A^{\prime}\right)$. This proves that $\bar{\mu}^{-1}\left(\overline{A^{\prime}}\right)=\bar{\alpha} \in \tau(\alpha)$ and $\tau(\alpha) \neq \varnothing$.

A halfring $H$ will be called monotypic if every nonzero ideal in $H$ is monotypic while $H[x]$ will be called class monotypic if every class in $G^{*}$ is monotypic.

3.5. THEOREM. If $H$ is a halfring, then $H$ is monotypic if and only if $H[x]$ is class monotypic.

Proof. Suppose $H$ is monotypic, $\alpha \in \mathcal{G}^{*}$ and $\beta \in \tau(\alpha)$. Then $\beta=\bar{\mu}^{-1}\left(B^{\prime}\right)$ for some $B^{\prime} \in \tau\left(A^{\prime}\right)=\left\{\overline{A^{\prime}}\right\}$. Hence $B^{\prime}=\overline{A^{\prime}}=(\bar{A})^{\prime}$ and it follows that

$$
\beta=\bar{\mu}^{-1}\left(B^{\prime}\right)=\bar{\mu}^{-1}(\bar{A})^{\prime}=\bar{\alpha} \text {. }
$$

Consequently, $\alpha$ is monotypic. Conversely, suppose $H[x]$ is class monotypic, $A^{\prime}$ is an ideal in $H$ and $\mu^{-1}\left(A^{\prime}\right)=\alpha \in \mathcal{G}$. If $B^{\prime} \in \tau\left(A^{\prime}\right)$, then $\bar{\mu}^{-1}\left(B^{\prime}\right) \in$ $\tau(\alpha)=\{\bar{\alpha}\}$ and it follows that

$$
\bar{\mu}^{-1}\left(B^{\prime}\right)=\bar{\alpha}=\bar{\mu}^{-1}\left(\overline{A^{\prime}}\right) .
$$

Consequently, $B^{\prime}=\overline{A^{\prime}}$ and $A^{\prime}$ is monotypic.

A halfring $H$ is called semisubtractive if for every $a, b \in H$ at least one of the equations $a+x=b$ or $a=b+x$ has a solution $x \in H$. It was shown in [4] that if $H$ is semisubtractive, then $H$ is monotypic.

3.6. Corollary. If $H$ is a semisubtractive halfring, then $H[x]$ is class monotypic.

Theorem 3.5 illustrates how a condition imposed on $H$ can influence $\mathcal{G} *$. 
Conditions imposed on $H$ can also influence the characterization of each class in $\mathcal{G}^{*}$. For example, suppose $H$ is Noetherian and $A$ is an ideal in $H[x]$. Since $\left\{A_{i}\right\}$ is an ascending chain in $H$, it follows that there exists $t$ such that $A_{i}=A_{t}$ for all $i \geqslant t$. Consequently, all the coefficients of terms of degree $i>t$ of a polynomial $f \in A$ lie in a fixed ideal $A_{t}$. This condition characterizes the class $\alpha=\mu^{-1}\left(A^{\prime}\right)$, i.e. $I \in \alpha$ if and only if there exists $q$ such that $I_{j}=A_{t}$ for all $j \geqslant q$. This proves the following.

3.7. Theorem. Let $H$ be a Noetherian halfring and $\mu^{-1}\left(A^{\prime}\right)=\alpha \in \mathcal{G}^{*}$. Then $I \in \alpha$ if and only if there exists $t \in Z^{+}$such that $I_{j}=A^{\prime}$ for all $j>t$.

3.8. EXAMPLE. Consider the halfring $Z^{+}$. Any nonzero $k$-ideal in $Z^{+}$is of the form $K=(a)$. Since $Z$ is a principal ideal ring, it is easy to see that $Z^{+}$is monotypic. Consequently, Theorem 3.5 assures that $Z^{+}[x]$ is class monotypic. Now $Z$ is Noetherian and Theorem 3.7 assures that if $B^{\prime}=(b)$ is an ideal in $Z$, then an ideal $I \in \beta=\mu^{-1}\left(B^{\prime}\right)$ if and only if there is a $t$ such that $I_{j}=B^{\prime}$ for all $j \geqslant t$. This implies that $b$ is the greatest common divisor of the set of all coefficients of polynomials in $I$. Consequently, each positive integer $n$ determines a class of ideals in $Z[x]$ and it follows that the number of classes in $Z[x]$ is equal to the cardinality of $Z$.

The above example may be extended to any halfring $H$ whose ring of differences $\bar{H}$ is a principal ideal ring.

\section{REFERENCES}

1. L. Dale, The $k$-closure of monic and monic free ideals in a polynomial semiring, Proc. Amer. Math. Soc. 64 (1977), 219-226.

2. __, Monic and monic free ideals in a polynomaial semiring, Proc. Amer. Math. Soc. 56 (1976), 45-50.

3. L. Dale and P. J. Allen, Ideal theory in the semiring $Z^{+}$, Publ. Math. Debrecen 22 (1975), 219-224.

4. H. Stone, Ideals in halfrings, Proc. Amer. Math. Soc. 33 (1972), 8-14.

Department of Mathematics, University of Alabama in Birmingham, Birmingham, Alabama 35294 